\title{
Robotic Connections: Customisable Joints for Timber Construction
}

\section{SIGRADI2018 TECHNOPOLITICAS \\ xxii congresso da sociedade iberoamericana de gráfica digital 22th conference of the iberoamerican society of digital graphics 07|08|09|novembro|2018 iau usp | são carlos | sp br}

\author{
Mikayla Heesterman \\ Victoria University of Wellington | New Zealand | mikayla.heesterman@gmail.com \\ Kevin Sweet \\ Victoria University of Wellington | New Zealand | kevin.sweet@vuw.ac.nz
}

\begin{abstract}
Timber is one of the most sustainable, renewable products, and coupled with computational tools has the potential to be redefined as a digital-age material. The research outlined in this paper employs contemporary digital fabrication techniques utilising a robotic arm to develop complex, CNC based parametric connections for engineered timber. While CNC joinery that utilizes three - five axis machining capabilities is increasingly common, the introduction of the six-axis robot as a machining tool provides greater freedom of movement and a wider range of complex procedures. This research returns to traditional Japanese timber craft, which offers unique structural and sustainable advantages. Using computational tools, new complex parametric connections suitable for contemporary fabrication will be designed and contribute to a library of joints suitable mass-customised in non-standard timber architecture.
\end{abstract}

Keywords: Robotics; Fabrication; Parametric; Timber; Architecture.

\section{INTRODUCTION}

\section{TRADITIONAL JAPANESE STRUCTURES}

The fundamental source of traditional Japanese architecture is its symbiotic relationship with nature, originating from their Shinto religion. This animalistic belief developed a style of architecture which aimed to coexist with the landscape and elements of Japan, particularly the abundance of wood (Locher, 2010). As such, traditional architecture became defined by an emphasis on visible wood structure as the primary feature (Futagawa \& Itō, 1967). The importance of displaying the timber strength and function through beauty, and the need to withstand natural disasters, led to high level of carpentry craftsmanship not seen elsewhere in the world. The splicing joints (connecting a continuous beam) and connecting joints (intersection between perpendicular elements) developed by craftsman often use small timber pins which hold the connection in place rather than any adhesives or fixings. These are inserted at low humidity which shrink the timber and use the expansion of the timber pieces to prevent the connection from coming apart. This material specific design works when all the proportions are carefully considered to maintain the natural strength ratio of the timber through considering bending, shear, torsion and compression (Sumiyoshi \& Matsui, 1989).

Combining this traditional knowledge with the development of engineered timber can revitalise an alternative structural system which has been largely abandoned. Steel and concrete structures rely upon rigid structure theory to be resilient; sufficient stiffness enables resistance against lateral forces. However, increased strength and stiffness further increases the earthquake action on the structure, in turn requiring more strength and stiffness in the structure to be resilient. This cycle can result in over-sized members, adding weight and size to a building and negatively affect its design. Contrastingly, traditional Japanese structures used flexibility rather than rigidity (Tanabashi, 1960). Intricately carved timber connections act as shock absorbers for earthquakes; the friction in the connections absorbs rather than resists lateral energy (Figure 1) (Seike, 1977). These designs accommodate the dynamic element of seismic actions, reducing the forces acting on the structure to move flexibly with an earthquake (Tanabashi, 1960). As well as intrinsic understanding of forces' behaviour, the structures' success relied upon craftsmen's material-specific knowledge which effect the structural integrity, such as species, location, size and position (Locher, 2010).

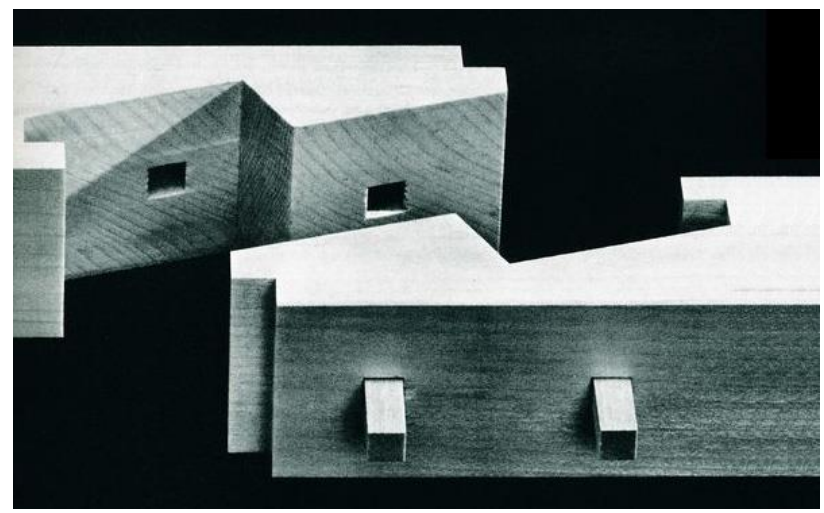

Figure 1: Mortised Rabbeted Oblique Splice (Seike, 1977)

Despite its successful resilience, this structural method has been abandoned with the gradual loss of craftsmanship and industrialisation's rise (Risatti, 2007). However, the 
advantages of engineered timber make it highly suitable for this traditional structural system, as its increased strengthto-weight ratio, consistency and size improve structural resilience. Its ability to be customised into complex, nonstandard components lends itself towards the intricately carved timber members (Mayo, 2015). Amalgamating this new material with traditional knowledge could facilitate a revitalisation of craftsmanship in order to advance contemporary timber use.

\section{ROBOTIC FABRICATION}

In comparison to older fabrication methods such as computer numerically controlled (CNC) milling, robotic fabrication is capable of significantly increased flexibility and complexity. This difference stems from the generic nature of industrial robotic arms, rather than task-specific CNC fabrication which is capable of a limited range of production (Dunn, 2012; Menges, 2012). While CNC milling is the most common fabrication method, robotic milling increases the possible complexity through a wider range of cuts and the ability to rotate around all axes. The shift from a five-axis maximum on a CNC machine, to a six-axis robotic arm (with additional axes available through a rotary table) removes cutting and rotation limitations, particularly for internal areas (Dunn, 2012). It also eliminates equipment constraints such as bed size, and permits completion of toolpaths in one, rather than multiple, stages. The fabrication applications of a generic robotic arm also drastically increase the opportunities for design-toproduction processes, where multiple tasks can be completed by one robot to achieve a sophisticated fabrication process. Using generative, computation methods with this technology allows efficient customization and diversity (Kocaturk, 2007). These software techniques improve the link between digital information modelling in CAD and production in CAM, increasing designer freedom and autonomy for creative non-standard architecture (Reffat, 2008).

\section{METHODOLOGY}

The project's methodology relied heavily upon a feedback loop between digital design and physical fabrication. Creating and testing in the virtual environment enabled ideas to be developed in the digital realm, using parametric software to create a robust customisable connection database. Testing these designs with physical robotic fabrication was more than a necessary step towards an outcome as it actively contributed to the development of the connections by adding material and machine based knowledge to the digital model. Testing began with relatively simple designs, with complexity slowly introduced - eventually leading to the design and production of new complex three-dimensional connections. The digitalphysical-digital process rebalances the hierarchy between conceptual and practical architecture, as both contribute equal but unique roles - redefining digital craftsmanship as a contemporary take on the master builder.

\section{A. TRADITIONAL PROPORTIONS}

The proportions and design of the joints reflect the tools used by the craftsmen. "The tool is the soul of the Japanese carpenter just as the sword was the soul of the samurai", and a traditional carpenter used approximately 180 different tools to create each complex connection (Locher, 2010, p. 63). These tools were extensions of their hands, which play a fundamental role in craft as the source of tacit understanding of materials. Although the project replaces traditional tools with new robotic technology, the original proportions still have an essential role. Sumiyoshi and Matsui's Wood Joints in Classical Japanese Architecture documents designs through dimensioned diagrams and instructions to explain the traditional methods and uses (Sumiyoshi \& Matsui, 1989). Reproduction of these joints requires maintaining a similar strength ratio in order to preserve the structural integrity of the design, several of which have been validated by modern structural analysis. Consequently, the dimensions were converted into proportions. Through analysis of the designs, each dimension was assigned a relationship with the $x, y$, or $z$ axis of the timber base (Figure 2). As the base size variates, the proportions ensure the dimensions change accordingly to ensure both the visual design and structural integrity are sustained. In some cases multiple sources provided conflicting information, which was addressed through creating the proportion as a parametric range rather than a static number. The effects of this were explored through the design phase.

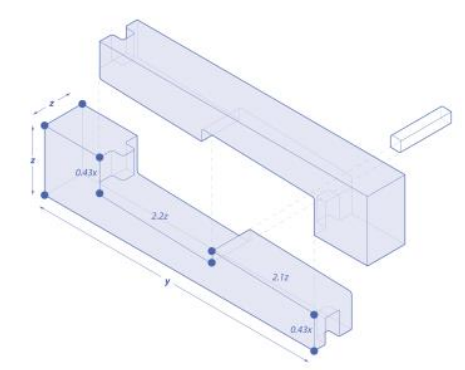

Figure 2: Traditional mortised model informed by proportions. Source: Authors.

\section{B. PARAMETRIC MODELS}

Following research into the design and construction of traditional joints, parametric digital models were developed in Grasshopper and Dynamo. These add-on software packages use visual programming language and nodebased editors which make scripting more accessible for architects (Ercan \& Elias-Ozkan, 2015). Parametric software allows the user to create relationships between geometries such as points, planes and lines from parameters, or variables, which inform or alter the design. Digital parametric methods allow efficient masscustomisation of different connections, based off both material conditions, fabrication limits and desired outcome. This contributed to the start of a fully customisable digital workflow, which enables both complexity and efficiency for non-standard architecture. There were two types of parameters used to create the connections; parameters relating to the conditions of the material - material-specific parameters, and what have been termed joint-independent parameters.

Material-specific parameters create a relationship between the size of the base timber, and the proportions of the design to ensure the connection is structurally resilient. Development of the digital models used the relationship between points as the primary parameter, as this allowed polylines and then solid geometry to be created. Points were moved from the origin, using the traditionally-informed proportions to create the three-dimensional shape of the connection. These points were then used to create solid 
geometry, which produced a visual representation of the digital connections. Therefore, as the dimensions of the timber base are changed, the relationship between points undergo a relative change to ensure the connection is appropriate for the size of the material. As a result, the connections are always structurally resilient, no matter the scale, as the ratio of strength is not compromised.

Joint-independent parameters were introduced to increase the level of customisation. While the dimensions are constrained and controlled by the timber base, independent parameters are not related to a specific external factor but rather chosen by the designer, although they are still limited by physical constructability. For example, the angle of a connection can range between $0^{0}$ $-180^{\circ}$ in order to create a bespoke connection, within the design limits of the script. As with all parameters, when the variable is changed the script instantly generates the newest iteration.

\section{TOOLPATH CREATION}

Traditional craftspeople worked to the limitations of their physical tools and factored these considerations into their design - such as avoiding curves which are difficult to create with chisels. Likewise, contemporary fabrication is constrained by its new tools, which result in different design limitations and potentials that need to be considered, such as the shape of the router and the order of the toolpath. This interdependent relationship between design and fabrication strengthens the autonomy of the process, resulting in better designs which take advantage of the fabrication system to improve the level of craftsmanship.

While CNC fabrication uses CAM software to automatically generate optimal toolpaths for objects, there is currently no interface between this and robotic software - due to the robot's generic nature (Dunn, 2012). Consequently, the robot's milling toolpaths had to be created through Grasshopper, which uses the add-on HAL Robotics to convert to proprietary Rapid Code, read by the robot. This caused a discrepancy between the digital connections modelled, which use solid geometry to create visual representations of the connections, and the Rapid Code which reads planes and moves the robot in a straight line between them. A script in Grasshopper was developed to extract planes from the solid geometry. For curves, this involved dividing the curve into a large number of planes to preserve the shape of the curve, while straight lines only required a plane at either end. The rotation of the plane was highly important as the spindle rotates accordingly, enabling it to achieve cuts with the side or end of the bit. Simulations created through HAL Robotics provided visual representation of the robot's movement through a toolpath, ensuring plane issues could be detected and resolved before fabrication.

As with CNC milling, robotic milling is constrained by the nature of the router bit, specifically its inability to cut square internal-corners. Consequently, in contrast to traditional craftsmanship which avoided curvature, contemporary fabrication must adjust angular designs to allow for filleted internal-corners. While this is often addressed in CNC milling through extending the internal corner (referred to as dog-boning), this results in excess gaps which compromises the visual design and structural integrity. Instead, connections were developed to translate them from a traditional to contemporary context. This often involved anticipating internal corners and ensuring the corresponding component would match. The intersection between two filleted edges often caused complex issues where the design was changed to ensure a clean cut. Throughout this the traditional connections remained as the fundamental design principles, and all modifications maintained the structural integrity.

The process of creating the toolpath accounted for factors automatically generated by CAM software, such as the depth and direction of passes and diameter of the router bit. To continue creating a customisable workflow, this process was fully parametric to allow the user control over the fabrication. Parameters included the diameter of the spindle which effected the diameter of filleted-corners, speed, RPM and the depth and direction of passes. Some of these parameters were joint-independent, such as the speed and RPM. Others were material-specific, for example the number of passes increased with the thickness of the timber. Building a system where every variable is a parameter increases the level of customisation of designs and creates more autonomy and intention over the fabrication. However, while traditional CAM software would be able to calculate the ideal combination, the process requires significant testing to achieve the optimal combination of these parameters to achieve a high-quality result.

\section{FULL-SCALE FABRICATION TESTS}

The fabrication process was an important part of the design feedback loop, providing insight into the connections rather than just a final product. Designing in a virtual environment limits understanding of physical properties, relationships and material behaviour (Gramazio \& Kohler, 2014). A continuous feedback loop between design and manufacture unifies the craftsman process and reveals new potentials that isolation of those processes would not expose. Fabrication revealed accuracy and material specific issues which the CAD model could not originally account for, but which could be re-calibrated into the digital design to improve it. After setting up an accurate environment, the simplicity of the $2 \mathrm{D}$ connections meant that fabrication testing was relatively quick, with minimal issues that needed resolving.

Before fabrication could begin, an accurate workspace had to be physically created and digitally modelled (Figure 3). Knowing the exact location of the work-surface that the timber is attached to allows a high level of precision in the milling. The workspace which contains the robot and worksurface (a low table) is uneven meaning the attached timber is not level. This was addressed by attempting to relevel the top of the table to reduce inaccuracies, however small bowing in the timber surface prevented it from being completely even. Using the robot, three points on the surface were located and their coordinates used to inform a plane modelled in Grasshopper. This slightly angled but accurate plane became the new coordinate system base used to create the parametric connections and toolpaths, rather than using the software world coordinate system. This resulted in significantly improved connections and greater control over the location of the timber. 


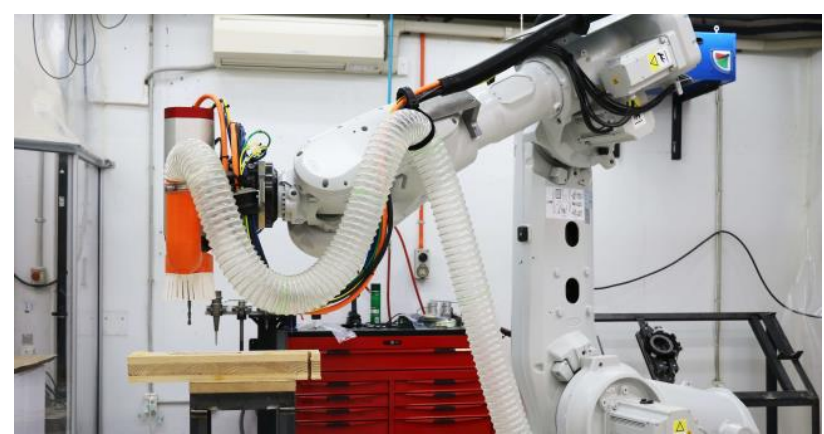

Figure 3: Robotic lab workspace with ABB IRB 6700 industrial arm with spindle attached. Source: Authors.

The accuracy of the robot (an ABB IRB 6700 industrial arm) is essential to any fabrication process. Industrial robotic arms are not predetermined to perform a specific task, consequently, each new end-effector (tool) had to be calibrated to a high level of precision in order to ensure accurate task performance. This was important in milling, as there is a small tolerance for connections to be successful and be neither too loose nor too tight which would prevent structural and aesthetic soundness. Early tests of the simple connections revealed consistent inaccuracies where the two sides of a joint would not fit smoothly or flush. These were a result of the imprecision of the TCP (tool centre point) of the particular bit being used. This necessitated recalibration of the bit, and every new bit as it was used, to increase the precision of the TCP to within $0.1 \mathrm{~mm}$ accuracy.

\section{RESULTS}

Using the described methodology a range of connections were designed and fabricated with an industrial robotic arm. These began with simple two-dimensional connections to reveal initial issues, and have worked up to a series of complex three-dimensional connections. Through this process new issues have been exposed and addressed in an iterative manner. Each design aimed to push the possibilities of robotic fabrication further, and explore the "potential of robot-induced design and materialisation processes" (Gramazio \& Kohler, 2014, p. 15).

\section{A. SIMPLE 2D CONNECTIONS}

Two simple traditional connections, a Dovetail and a Mitred-Finger joint, were selected to begin testing digital and physical fabrication (Figure 4). The simplicity of these connections meant there was little design adaptation required to prepare them for fabrication, allowing the focus to be on testing the new digital and physical workflow.

While the dovetail was limited by its simplicity, the MitredFinger joint presented an opportunity to use a jointindependent parameter to test how robust the script was. The angle of the connection between the two components of the joint was constrained between $20^{\circ}-160^{\circ}$. This was further restrained by the limitation of constructability, resulting in a parameter ranging between $60^{\circ}-120^{\circ}$. This parameter can be changed by the user to produce a new digital model with the corresponding angle, depending on the type of connection desired. Several different angles were successfully modelled and fabricated as evidence of the success of the digital script.
The simplest connection, the Dovetail, was easily adapted by filleting the corners. This was parametrically dependent on the diameter of the router bit, so the rounded corners were no larger than necessary. In contrast to this, the Mitred-Finger required no design modifications, but the toolpath had to be designed in a particular order to ensure no inadvertent rounded edges were created. Both designs required consideration for the effect of the spindle diameter on the shape of the two sides of the connection. Each half of the Dovetail connection had a different shaped toolpath which had to be cut separately, to allow for the spindle diameter.

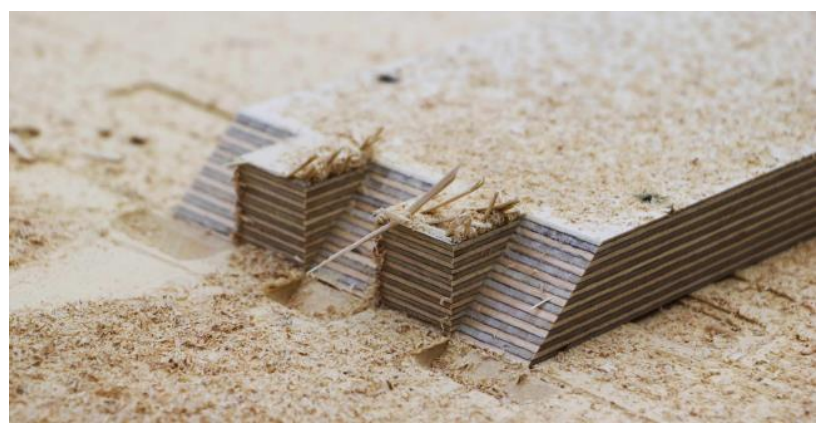

Figure 4: Fabrication testing of Mitered-Finger Connection.

$$
\text { Source: Authors. }
$$

Initial testing through fabrication exposed material specific issues for timber, such as rough edges. Multiple iterations of the Dovetail and Mitred-Finger had small lips on the inside or feathered edges. Different variables were systematically changed and tested, including altering the depth and direction of passes, speed and extracting more sawdust. The issue was resolved through lowering the drilling speed from 20,000 RPM to 16,000 RPM (whilst moving at $30 \mathrm{~mm} / \mathrm{s}$ ). This reduced the frequency of the spindle and prevented chatter - a resonant phenomenon causing the worksurface to vibrate slightly. Fixing this issue produced consistently well-fitting Dovetail and Mitred-Finger connections that act as initial proof of concept for robotic milling.

\section{D CONNECTIONS CRITICAL ANALYSIS}

Although the project aimed to focus on complex threedimensional joints, the two-dimensional connections successfully acted as proof of concept for both the digital parametric toolpath, and the potential of robotic milling fabrication. Beginning with these simple designs allowed issues to be easily identified and resolved before more complexity was added.

\section{B. 3D CONNECTIONS: MORTISE SPLICE}

Several complex traditional Japanese timber-only connections were researched and parametrically modelled, such as the Mortised Rabbeted Oblique Splice. These interlocking splices with draw pins were commonly used for beams, and testing shows high tensile resilience (Sumiyoshi \& Matsui, 1989). The initial iteration of Mortise Splices began with the traditional design, however, with each iteration the design deviated from the original. While the aesthetics of the connections changed, subsequent iterations still relied upon the traditional proportions of the original Mortise splice. This ensured the strength ratio was maintained, despite significant design changes. Although these design changes mean that new structural testing would be required, retaining the traditional proportions provides a structural limitation to the design. 
The multi-dimensional nature of the connections required significant design work to adapt the traditional connection to suit contemporary fabrication limitations. While the Dovetail used filleted-corners to account for the router bit, this simple solution alone would not achieve the complex shape - which requires milling both vertically and horizontally. The introduction of horizontal milling created issues at the junction between two perpendicular filleted corners, where the spindle was unable to cut the complex 3D curve due to the diameter of the spindle. This particularly effected the tenon of the traditional Mortised Splice, resulting in differences between the inverse shape. This demonstrated the differences between traditional and contemporary fabrication, and subsequently initiated design developments which suit contemporary fabrication while still maintaining the key traditional principles. This involved altering the end tenon to ensure it could be successfully milled in one stage while still achieving its role of preventing vertical movement of the joint. Several iterations were designed and tested; such as a curved tenon, an asymmetric tenon, a V-shaped tenon and a stepped-back tenon. The combination of the stepped-back tenon and the curved tenon provided a successful solution to the technical issue. While the iterations were primarily technically driven as a response to toolpath issues, design decisions also impacted the series. The final iteration was a simplified and stylised development of the iterations, with an emphasis on curved components (Figure 5). This begins to work to the advantages of robotic fabrication, which can easily create smooth curves, rather than the limitations of traditional methods.

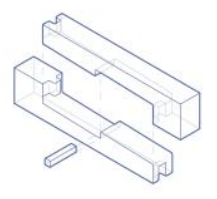

(1)

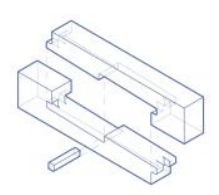

(2)

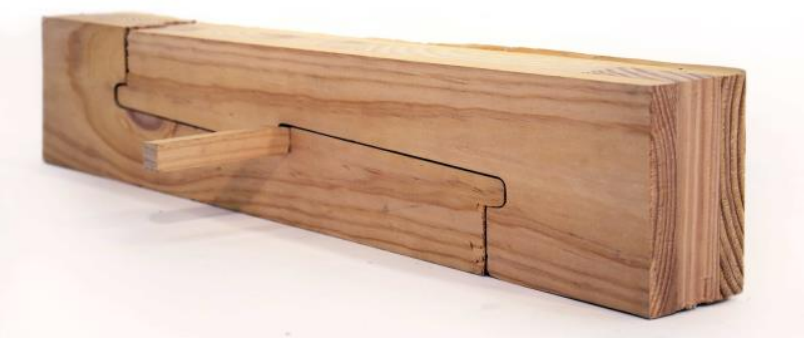

Figure 5: Diagrams of three iterations of Mortise Splices \& fabrication tests. Source: Authors.

The added complexity of the connections required the spindle to approach from multiple different directions and rotations - requiring a more robust system to correctly rotate toolpath planes. A small script, or Grasshopper cluster, was developed which allowed planes to be rotated to the correct orientation using the optimal position of the robot and the work-surface coordinate system. Consideration was taken to ensure the rotation of each of the six joints was possible, as although they can rotate $360^{\circ}$, they are physically limited by cables attached to the arm. This was particularly important when moving between planes of two different rotations, as often the most efficient joint movement calculated by the robot would cause cables to become stretched or tangled. To account for this issue a 'home position' was utilised between large changes in rotation, and at the beginning and end of a toolpath. The 'home position' returns the arm to a neutral and known location and rotation, ensuring the following movements rotate the arm as intended in the simulations. A singularity command was also inserted into the script, preventing the robot from rotating into singularity (an impossible position). Another Grasshopper cluster was created to automatically add clearance planes in a chosen direction to a section, allowing the spindle to clear the work-surface before significant direction or rotation change. These toolpath developments ensure that the increase in complexity is met with an equally parametric toolpath, which is able to mill the timber in a single path, using rotation not possible with a five-axis router.

The complexity increased the range of rotation undertaken by the robotic arm, requiring a new work-surface to accommodate this. The previous work-surface was a low, $1.0 \mathrm{~m} \times 0.6 \mathrm{~m}$ table, which prevented the spindle from approaching a timber piece from more than two sides (when positioned at the corner) without colliding with the table. Consequently, a new $0.15 \mathrm{~m} \times 0.3 \mathrm{~m}$ table raised $0.5 \mathrm{~m}$ above the original table was developed to accommodate the need to approach from at least three sides, and the top. This is the width of the timber being milled, ensuring the robot is able to rotate around the entire table and mill without the spindle hitting the table. This allowed a wider range of complex connections to be fabricated, particularly connections involving horizonal milling from more than one direction.

Testing through fabrication exposed more material specific issues for timber that were not relevant for the simple designs, such as the tolerance level of the cuts. The simplest connections did not require any tolerance consideration, due to the spindle remaining on the vertical axis. However, complex connections are required to fit several interlocking elements simultaneously, and a slight inaccuracy can prevent the entire joint from working. Fabrication tests of multidimensional connections revealed small inaccuracies in the location of the spindle on horizontal or angled cuts. This originates from small TCP (tool centre point) inaccuracies that remain despite multiple recalibrations. In response TCP testing was undertaken, and a specific tolerance for each direction was discovered. This is scripted back into the digital model to produce highly accurate physical models - made possible by the digital-tophysical feedback loop. Ideally, the calibration issue would be resolved, as currently each joint must be individually tested to discover and accommodate the specific tolerance issues.

\section{MORTISE SPLICE CRITICAL REFLECTION}

The Mortise Splice introduced the complexity of a threedimensional connection and addressed the resulting issues through a series of iterations. The decisions were predominantly technically driven, rather than structural or aesthetic, due to the toolpath issues that arose. Some design decisions were made which demonstrate beginning to work to the parameters and strengths of the robot, rather than remain limited by traditional thinking and methods. While the flexibility of the six-axes robot was used to produce three-dimensional joints, exploring specifically robot-induced design would further expand the potential of timber connections. 


\section{3D CONNECTION: CURVED SPLICE}

Reflecting on the process of the Mortise Splice highlighted the need for the research to shift beyond simply recreating traditional designs, and instead focus on developing new connections. A series of Curved Splices were developed, which explored the ability of the robot to produce complex curves which were not possible to create with traditional tools and methods.

The Curved Splice was developed as a new contemporary design which is directly informed by the proportions of the Mortise Splice, while introducing concepts not used in traditional joinery such as curves. Instead of the traditional angular splice and pin, it uses an ' $S$ ' shape arc with a slight curvature in the middle. This allows the connections to be pressed together, clicking into place as the two elements align. The friction of the curvature prevents the elements from being easily pulled apart, due to the widening at the ends. The proportions of the S-shaped curve are identical to the proportions of the traditional Mortise Splice. As with Japanese connections, the design prevents movement in the three different axes through mortise and tenon elements. The design also utilises a contemporary development of the traditional mortise and tenon at the ends of the ' $S$ ' curve to prevent the connection from sliding sideways. The resulting design is a complex threedimensional connection which is highly suitable for robotic fabrication, and requires the six axes to be produced (Figure 6). The use of traditional proportions to inform the curvature sustains the strength ratio and the structural resilience of the design. Furthermore, the curvature of the design lowers the stress concentration of the connections.

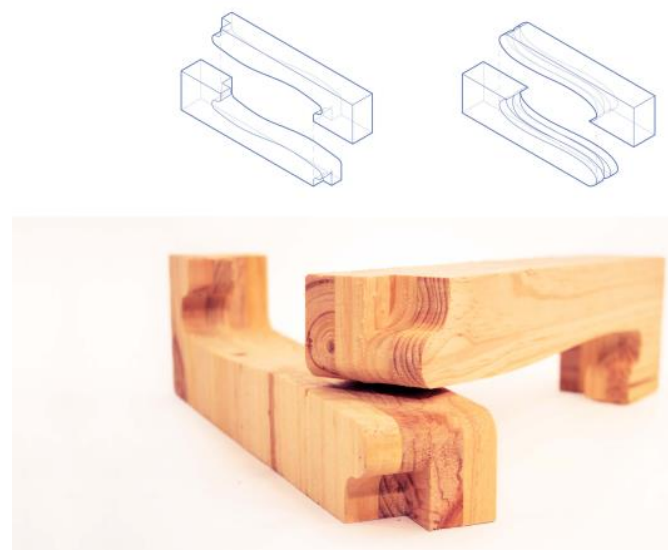

Figure 6: Diagrams and model of Curved Splice iterations. Source: Authors.

The introduction of curves caused a change in the way toolpaths planes were created. Previously, all planes on a single line were uniformly aligned with an axis. However, the Curved Splice required the bit to change rotation as it travelled along a curve, resulting in planes that were perpendicular to the direction of the curve. This caused issues with the rotation of the robotic arm's axis, as the arm often underwent significant changes in a small amount of time. This was addressed through realigning the perpendicular planes to a normal - ensuring they were perpendicular to the curve but uniformly aligned in the other axes.

The increase in complexity often required milling to be primarily completed on the horizontal axis rather than the vertical axis. This necessitated the introduction of tabs to prevent the top piece from falling down onto the spindle while it was horizontally milling. A Grasshopper cluster was developed which parametrically created the tabs at a given location. The user has full control over the size, depth, number, and location of the tabs to ensure it is as customisable as the rest of the system.

The Curved Splice iterations used friction and the shape of the curve to hold the connections together, which required significant testing to achieve the optimal curvature. Initial tests began with a curvature of $1.5 \%$, which was gradually increased through iterations to $10 \%$ to reveal an optimal curvature of $6 \%$. This was able to be put together while providing enough friction to prevent the connection from being pulled apart. This demonstrates the feedback loop between digital and physical fabrication, as physical testing revealed what digital modelling could not. The two sides of the method were successfully integrated to improve the overall design.

The Curved Splice iterations required the robotic arm to turn the spindle upside down in order to cut one of the complex curves (Figure 7). This demonstrated the full use of the six axes of the robot to achieve a cut not possible with a standard CNC machine. Although Grasshopper and HAL Robotics provides a visual simulation of the toolpath, this is limited to showing the robots position at each plane. However, it is often the movement between planes that cause the cables to tangle, or the robot to reach singularity. Therefore, for such complex designs the toolpaths were physically tested before the spindle was attached.

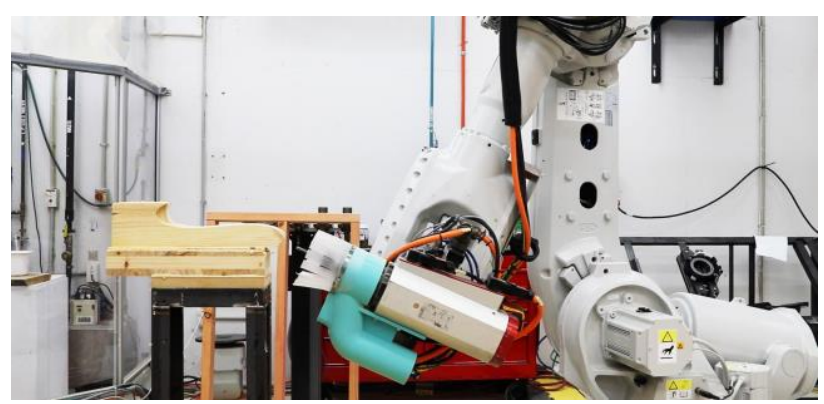

Figure 7: Rotation of sixth axis of robotic arm. Source: Authors.

\section{CURVED SPLICE CRITICAL REFLECTION}

The Curved Splice iterations pushed the use of the robot beyond what is possible with other milling machines in order to create complex interlocking connections. Unlike previous designs, the curved shape is highly suitable for robotic fabrication and uses this contemporary technology to its advantage. This 'robot-induced' connection utilised the physical fabrication process to improve the design, rather than use it as the final means for fabrication. However, the design is still highly informed by the technical capabilities of the robot and the structural requirements of the traditional proportions. More emphasis on aesthetic decisions is needed to raise the level of craftsmanship of the connection.

\section{3D CONNECTIONS: LOCKING THROUGH MORTISE}

As several series of splices had been explored, a perpendicular connection was developed. This began with researching a Through Mortise connection and used the traditional proportions to inform the structural design. Reflecting on the previous series revealed the need to push 
the aesthetic design further in the effort to create a highly crafted connection. This was done in conjunction with creating a design that took advantage of the robot's flexibility and ability to mill complex shapes. The resulting iterations experiment with chamfered curved tenons which slots into a column with carvings that reflect the internal shape of the tenon (Figure 8). A locking pin is inserted and turned to prevent the joint from coming apart.
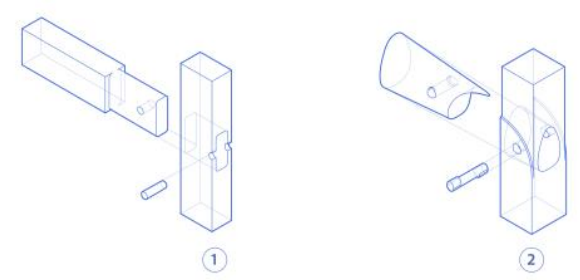

Figure 8: Locking-Through Mortise iterations. Source: Authors.

The Locking-Through Mortise added new complexities which effected the toolpath creation and fabrication. Both the beam and the column component required multiple passes; a roughing and a finishing pass. The roughing pass removes large amounts of material before the finishing pass mills the detailed components. Integrating these into the toolpath creation increased the efficiency of the milling, as the roughing pass can quickly prepare the timber for detailed fabrication.

The fabrication process instigated the use of the bit and spindle in new innovative ways to solve problems and inefficiencies. The size of the components necessitated the use of a large $20 \mathrm{~mm}$ diameter, $220 \mathrm{~mm}$ long bit. This was used on its side for the first time to achieve the curved chamfered tenon component and create a smooth curved finish. Trials with this fabrication technique revealed specific limitations to working with the spindle on its side, such as the need for smaller passes to avoid the creation of small ridges. Several toolpaths involved using the spindle completely upside down. Issues had arisen when roughing a deep hole in the column that caused sawdust to pile in the hole and catch on fire. After exploring different pass depths and pausing the program for dust extraction, the toolpath was flipped upside down to cut the hole from underneath (Figure 9). This proved very effective at allowing the saw dust to instantly fall away from the component. The key issue with using the spindle upside down was the clearance between the spindle body and the work surface table. The base timber had to be positioned on the work surface with a significant overhang to allow room for the spindle to move freely alongside the table.

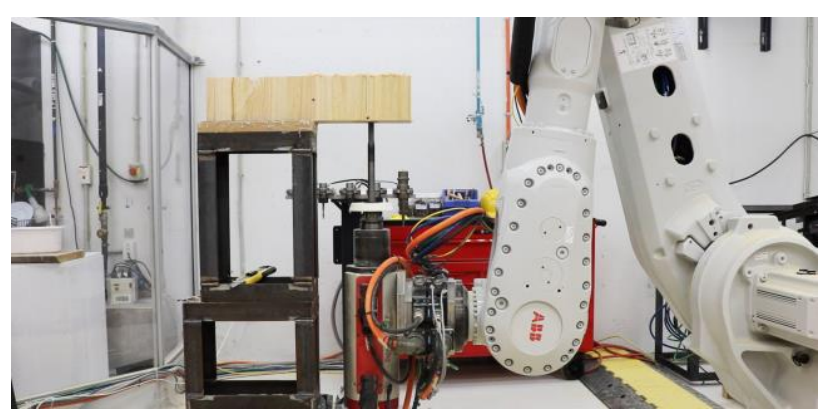

Figure 9: Upside down rough-cut to prevent saw dust heating up. Source: Authors.
Unlike the splice series, the Locking-Through Mortise required several components to slide into one another; the beam slots into the column and the pin slots into the beam. This presented tolerance issues where components would not fit. Through fabrication testing several optimal tolerances were discovered. Small components require $0.5 \mathrm{~mm}$ tolerance, whereas large components need up to $1 \mathrm{~mm}$. Currently, this solution is not fully parametric, as each relationship between components needs to be physically tested for the ideal tolerance. This is exacerbated by the slight TCP inaccuracies discussed above.

\section{LOCKING-THROUGH CRITICAL REFLECTION}

The Locking Through Mortise integrated a better balance of design, structural and technical decisions to create new connections. The iterations have explored complex curves to push the potential of the robot and test its flexibility. Through this process new digital and physical issues have been approached through solutions that are only possible with the robotic technology available. Where traditional machinery would struggle, the robot allows innovative problem solving which has the opportunity to advance the design rather than hinder it.

\section{E. DIGITAL CONNECTION DATABASE}

Through this process several series of connections have been produced; the Mortise Splice, Curved Splice and Locking-Through Splice. However, the design and fabrication of complex joints are not in isolation, but have led towards the creation of a digital connection library. This database of different successful connections aims to become fully accessible to other designers and architects. The rigorous testing that each connection has undergone refines the design and fabrication to the point where the joint is structurally resilient, aesthetically pleasing, and can be successfully fabricated by a six-axis robotic arm. In addition, each joint is fully customisable through the use of various parameters - either dependent on the material, the bit, or independent. This creates a high level of flexibility, where the system responds to different timber sizes, bit sizes, proportions or aesthetic requirements. Through the process developed, individualised toolpaths are generated without any interaction with the Grasshopper script, ensuring the process is accessible to people who are not familiar with the software. This library of connections is enhanced by information about each connection, such as joint classification, typical use, resilience and strength ratio.

\section{CONCLUSIONS}

\section{KNOWLEDGE CONTRIBUTION \& LEARNING}

Robotic fabrication and computational tools has opened up the opportunity for a new interpretation and greater potential for material-specific designs which have been slowly abandoned. The flexibility of robotic fabrication allows traditional joinery to be accessible again and increases its scope through the new opportunities that this technology creates. While traditional craft was replaced with easier to produce materials and methods, returning to them from a computational perspective allows a database of customisable multidimensional joints to be created. While these are rooted in traditional concepts, they move beyond this knowledge through adapting their concepts to become more suitable for contemporary fabrication, such as the use of curves. This development improves their 
suitability for contemporary non-standard architecture, and has the potential to be more structurally resilient when analysed with finite element modelling. As the research continues, it will focus on taking better advantage of the robots to drive and inform how the joint design develops.

Critical reflection of the research reveals several challenges. A lot of the iterations were driven by technical decisions to ensure the physical fabrication worked, such as sharp corners and internal areas. Fabrication testing took significantly more time as each individual issue took several iterations to identify and resolve. Further research will aim to give more weight to aesthetic design decisions alongside fabrication and structural needs. These challenges highlighted the need to equally balance the digital and physical sides of architecture to create a process which produces craft. Fabrication limitations and potentials should become a consideration during the design phase, positively impacting and improving the process. Tolerance is an ongoing issue which disrupts the digital workflow as the tolerance for each joint needs to be calculated. However, a digital database will be able to have the calculated tolerance built back into the script.

While the feedback loop allows for the material to be considered, a fully material specific process is limited due to the anisotropic nature of timber. Unlike traditional craftsmanship, individual timber irregularities -which exist even in engineered timber - affect both the aesthetic and structural integrity of the design and cannot be scripted into a digital toolpath. Subsequently, there remain parts of the fabrication process which prefer human intelligence over scripted models to ensure fibre continuity and milling direction of the timber is correct to maintain structural resilience.

\section{FUTURE WORK}

While this research is highly relevant, future steps will aim to increase the suitability and accessibility for the industry in order to become a new connection solution. The scope will be expanded to include a wider range of connections and uses; varying from structural to non-structural, beams, columns, splices and sheet joints. Locking pins that can be cut or 3D printed will also be investigated. This expansion of the connection database will enable the consideration of a wider system, rather than focusing solely on an individual connection. This will facilitate larger scale components to be designed cohesively - such as complex post and beam structural systems utilising multiple customisable connections. This further research will allow connections to become an element of the design, rather than only a structural restriction. The ability for these to be easily customised and fabricated with increased complexity makes then highly suitable for the engineered timber industry, which is often used for non-standard architecture.

Other considerations to be addressed include improving the interface between commonly used industry products such as Revit and robotics. The Revit add-on Dynamo was successfully used to develop the parametric milling toolpaths, however there is currently not an interface between this and ABB robots. Improving this software discrepancy will increase the accessibility of new types of fabrication and lift this research from the lab to the construction industry.

Partnering with structural engineers will allow finite element modelling of the connections, revealing weaknesses which can be improved. This would align with current structural theory and validate new designs - such as the Curved Splice which is expected to reduce stress. Structural validation will facilitate this research becoming a largescale resilient structural method, and help the potential of robotic fabrication to be realised in the construction industry.

\section{REFERENCES}

Dunn, N. (2012). Digital Fabrication in Architecture. Computer (Vol. 45). https://doi.org/10.1007/s00004-012-0130-8

Ercan, B., \& Elias-Ozkan, S. T. (2015). Performance-based parametric design explorations: A method for generating appropriate building components. Design Studies, 38, 33-53. https://doi.org/10.1016/j.destud.2015.01.001

Futagawa, Y., \& Itō, T. (1967). The essential Japanese house; craftmanship, function, and style in town and country. Tokyo: J. Weatherhill.

Gramazio, F., \& Kohler, M. (2014). Made by Robots : Challenging Architecture at the Large Scale AD. Architectural Design (Vol. 84). London: John Wiley \& Sons, Inc. https://doi.org/10.1002/ad.1752

Kocaturk, T. (2007). An Investigation of the Emerging Knowledge in Digital Design from a Multidisciplinary Perspective. Architectural Engineering and Design Management, 3(2), 93105.

Locher, M. (2010). Traditional Japanese architecture an exploration of elements and forms. Tokyo: Tuttle.

Mayo, J. (2015). Solid wood: case studies in mass timber architecture, technology and design. New York: Routledge.

Menges, A. (2012). Material resourcefulness: Activating material information in computational design. Architectural Design, 82(2), 34-43. https://doi.org/10.1002/ad.1377

Reffat, R. M. (2008). Digital Architecture and Reforming the Built Environment. Journal of Architectural and Planning Research, 25(2), 118-129.

Risatti, H. A. (2007). A theory of craft: function and aesthetic expression. Chapel Hill: University of North Carolina Press.

Seike, K. (1977). The Art of Japanese Joinery (1st Englis). New York: J. Weatherhill.

Sumiyoshi, T., \& Matsui, G. (1989). Wood Joints in Classical Japanese Architecture. Japan: Kajima Institute of Publishing.

Tanabashi, R. (1960). Earthquake Japanese Resistance of Traditional. Disaster Prevention Research Institute, Kyoto University, 40, 15. Retrieved from http://repository.kulib.kyotou.ac.jp/dspace/bitstream/2433/123698/1/b40p0n000p01.pdf 